\title{
A General Formula of Polynomial Interpolation.
}

\author{
By A. C. Aithen. \\ (Received 2nd March 1929. Read 3rd May 1929.)
}

§1. Enuncration and Proof.

The operations ${ }^{1}$ of differentiation, ordinary and central differencing, divided differencing, and forming linear combinations of the results of these, have in common the distributive, associative and commutative properties, and the further property that when performed on a general polynomial of the $n^{\text {th }}$ degree they produce a polynomial of the $n-1^{\text {th }}$ degree, while when performed on a constant they yield zero.

Consider $n$ explicitly defined operators $\theta_{1}, \theta_{2}, \theta_{3}, \ldots \theta_{n}$, possessing all of these properties, except possibly the commutative with each other. The question of any numerical factor in $\theta_{r}$ may be settled without loss of generality by assuming $\theta_{i}, x=1$, and we further assume that the effect of the inverse operation $\theta_{r}^{-1}$ on a polynomial is determinate except for an arbitrary added constant. In accordance with non-commutative algebra we shall have

$$
\left(\theta_{r} \theta_{r-1} \ldots \theta_{1}\right)^{-1}=\theta_{1}^{-1} \theta_{2}^{-1} \ldots \theta_{r-2}^{-1} \theta_{r}^{-1} \text {. }
$$

The problem now proposed is this: $f(x)$ is a polynomial of the $n^{\text {th }}$ degree, and we are given the numerical values of

$$
f(x), \quad \theta_{1} f(x), \quad \theta_{2} \theta_{1} f(x), \ldots, \theta_{n} \theta_{n-1} \ldots \theta_{1} f(x),
$$

for $x=0$, or alternatively for $x=a$. It is required to determine $f(x)$ from these values. If by convention we write, e.g. the value of $\theta_{2} \theta_{1} f(x)$ for $x=a$ as $\theta_{2} \theta_{1} f(a)$, then the formula required is

$$
\begin{array}{r}
f(x)=f(0)+x \theta_{1} f(0)+\theta_{1}^{-1} x . \theta_{2} \theta_{1} f(0)+\left(\theta_{2} \theta_{1}\right)^{-1} x . \theta_{3} \theta_{2} \theta_{1} f(0)+\ldots \\
+\left(\theta_{n-1} \ldots \theta_{1}\right)^{-1} x . \theta_{n} \ldots \theta_{1} f(0), \ldots \ldots \ldots \ldots(1)
\end{array}
$$

where the polynomials $\theta_{1}^{-1} x,\left(\theta_{2} \theta_{1}\right)^{-1} x, \ldots$ have their arbitrariness removed by the conditions that $\left(\theta_{r} \theta_{r-1} \ldots \theta_{1}\right)^{-1} x$ is such that

1 Cf. Dr J. F. Steffensen's Interpolation (Baltimore, 1927), pp. 178, 184. 
$\theta_{r}^{-1} x, \quad\left(\theta_{r} \theta_{r-1}\right)^{-1} x, \ldots\left(\theta_{r} \cdot \theta_{r-1} \ldots \theta_{1}\right)^{-1} x$ vanish simultaneously for $x=0$.

Proof. Suppose that the theorem (1) is true for the polynomial $\theta_{1} f(x)$ as obtained from $\theta_{1} f(0), \theta_{2} \theta_{1} f(0), \ldots$, that is, suppose

$$
\begin{array}{rl}
\theta_{1} f(x)=\theta_{1} f(0)+x \theta_{2} \theta_{1} f(0)+\theta_{2}^{-1} & x . \theta_{3} \theta_{2} \theta_{1} f(0) \\
& +\left(\theta_{3} \theta_{2}\right)^{-1} x . \theta_{1} \theta_{3} \theta_{2} \theta_{1} f(0)+\ldots .
\end{array}
$$

Operating on this with $\theta_{1}^{-1}$, since $\theta_{1}^{-1} \mathrm{l}=x$, and

$$
\theta_{1}^{-1}\left(\theta_{r} \theta_{r-1} \ldots \theta_{2}\right)^{-1}=\left(\theta_{r} \theta_{r-1} \ldots \theta_{1}\right)^{-1} \text {, }
$$

we have

$$
f(x)=C+x \theta_{1} f(0)+\theta_{1}^{-1} x \cdot \theta_{2} \theta_{1} f(0)+\left(\theta_{2} \theta_{1}\right)^{-1} x . \theta_{3} \theta_{2} \theta_{1} f(0)+\ldots
$$

Putting $x=0$ and using conditions (1a) we see that $C=f(0)$. Hence the validity of the theorem for $f(x)$ depends on its validity for $\theta_{1} f(x)$, which similarly, under conditions (1a), may be proved to depend on its validity for $\theta_{2} \theta_{1} f(x)$, and so on. But it is obviously true for $\theta_{u} \theta_{n-1} \ldots \theta_{1} f(x)$, for since this is a constant, we must have

$$
\theta_{n} \theta_{n-1} \ldots \theta_{1} f(x)=\theta_{n} \theta_{n-1} \ldots \theta_{1} f(0) .
$$

Hence, step by step, the theorem is true generally.

A useful alternative form of the theorem is

$$
\begin{array}{r}
f(x)=f(a)+(x-a) \theta_{1} f(a)+\theta_{1}^{-1}(x-a) \theta_{2} \theta_{1} f(a) \\
+\left(\theta_{2} \theta_{1}\right)^{-1}(x-a) \theta_{3} \theta_{2} \theta_{1} f(a)+\ldots .
\end{array}
$$

the polynomial coefficients being in this case determined by the conditions that $\left(\theta_{r} \theta_{r-1} \ldots \theta_{1}\right)^{-1}(x-a)$ is such that

$$
\theta_{r}^{-1}(x-a), \quad\left(\theta_{r}, \theta_{r-1}\right)^{-1}(x-a), \ldots\left(\theta_{r} \theta_{r-1} \ldots \theta_{1}\right)^{-1}(x-a)
$$

vanish simultaneously for $x=a$.

\section{§2. Applications of the Formula.}

(i) Let $\theta_{1}=\theta_{2}=\ldots=\theta_{n}=D$, the operation of differentiating with respect to $x$. Then $D^{-1} x=x^{2} / 2$ !,$D^{-2} x=x^{3} / 3$ !, etc., so that formula (1) is the Maclaurin expansion, (2) the Taylor expansion of $f(x)$. 
(ii) Let $\theta_{1}=\theta_{2}=\ldots=\theta_{i n}=\Delta$, where $\Delta \phi(x)=\phi(x+1)-\phi(x)$. Then, by the properties of factorial polynomials,

$$
\Delta^{-1} x=x(x-1) / 2 !, \quad \Delta^{-2} x=x(x-1)(x-2) / 3 !, \text { etc. }
$$

so that (1) becomes the Gregory-Newton formula of interpolation.

(iii) Let the given values be

$$
f(0), \Delta f(0), \Delta^{2} f(-1), \Delta^{3} f(-1), \Delta^{4} f(-2), \Delta^{5} f(-2), \ldots
$$

Then $\theta_{1}=\Delta, \theta_{2} \theta_{1}=\Delta^{2} E^{-1}, \theta_{3} \theta_{2} \theta_{1}=\Delta^{3} E^{-1}, \theta_{4} \theta_{3} \theta_{2} \theta_{1}=\Delta^{4} E^{-2}, \ldots$ and we have at once a set of factorials satisfying conditions (1a),

$$
\begin{array}{r}
\theta_{1}^{-1} x=x(x-1) / 2 !,\left(\theta_{2} \theta_{1}\right)^{-1} x=(x+1) x(x-1) / 3 !, \\
\left(\theta_{3} \theta_{2} \theta_{1}\right)^{-1} x=(x+1) x(x-1)(x-2) / 4 !, \ldots
\end{array}
$$

These are the well known coefficients in the Newton-Gauss formula of interpolation, which uses the particular differences here specified.

At this point a remark may be made, of general application to interpolation by finite differences. Almost all of the formulae in common use involve differences of ascending order which are also adjacent, that is, attained each from the preceding by a single downward or upward oblique step in the table of differences. Now the operation corresponding to such a step is $\Delta$ or $\Delta E^{-1}$, and so to a succession of such steps is $\Delta^{r} E^{-s}$, with $r \geqslant s$. But $\Delta^{-r} E^{s} x$ is the factorial $(x+s)(x+s-1) \ldots(x+s-r) /(r+1) !$, which for $r \geqslant s$ contains $x$ as a factor and so vanishes for $x=0$. It follows that the conditions (la) will be satisfied in whatever order we perform the inverse operations. We may indeed completely reverse the order, in which case a study of any two consecutive terms, say $\theta_{2}^{-1} \theta_{1}^{-1} x . \theta_{3} \theta_{2} \theta_{1} f(0)$ and $\theta_{3}^{-1} \theta_{2}^{-1} \theta_{1}^{-1} x . \theta_{4} \theta_{3} \theta_{2} \theta_{1} f(0)$, shows that the new operator $\theta_{3}$ affecting $f(0)$ in the first term is answered by its inverse $\theta_{3}{ }^{-1}$ affecting the polynomial coefficient in the second term. On this fact, and on the properties of factorials under the operations $د^{-1}, د^{-1} E$, those rules depend which have been given ${ }^{1}$ for attaching the proper coefficients to differences lying in a prescribed route across a table.

1 e.g. by W. F. Sheppard in the article on Interpolation, Encyc. Brit., 11th Edition, 1910, Vol. XIV., p. 710, 
When the successive differences used do not proceed by adjacent oblique steps but by wide skips, the factorial polynomials obtained by the inverse operations do not contain $x$ as a factor but must be affected by an added constant in order to vanish with $x$. The general formula (1) enables us, however, to write down readily the proper coefficients, provided we attend strictly to the order of inverse operations.

Example. Given $f(0)=1, \Delta f(2)=25, \Delta^{2} f(3)=26, \Delta^{3} f(1)=6$, find the cubic $f(x)$.

Here $\theta_{1}=\Delta E^{2}, \theta_{2}=\Delta E$, and we have at once, by means of factorials and (1a),

$$
\begin{aligned}
\left(\Delta E^{2}\right)^{-1} x & =(x-2)(x-3) / 2 !-3=\left(x^{2}-5 x\right) / 2, \\
\left(\Delta E^{2}\right)^{-1}(\Delta E)^{-1} x & =\left(E^{2}\right)^{-1}[(x-1)(x-2) / 2 !-1] \\
& =(x-3)(x-4)(x-5) / 3 !-10-x \\
& =\left(x^{3}-12 x^{2}+41 x\right) / 6 .
\end{aligned}
$$

Hence $f(x)=1+25 x+13\left(x^{2}-5 x\right)+x^{3}-12 x^{2}+41 x$

$$
=x^{3}+x^{2}+x+1 \text {. }
$$

(iv) Let $\theta_{r} \phi(x)=\left[\phi(x)-\phi\left(a_{r}\right)\right] /\left(x-a_{r}\right)$, that is, the operation of divided differencing. Then $\theta_{1} f\left(a_{0}\right), \theta_{2} \theta_{1} f\left(a_{0}\right), \ldots$ are identical with the successive divided differences usually denoted by $f\left(a_{0}, a_{1}\right), f\left(a_{0}, a_{1}, a_{2}\right), \ldots$ Also the operations $\theta_{1}, \theta_{2} \theta_{1}, \ldots$ performed on $\phi(x) \equiv\left(x-a_{0}\right)\left(x-a_{1}\right) \ldots\left(x-a_{n}\right)$ are equivalent to ordinary division by $\left(x-a_{1}\right),\left(x-a_{1}\right)\left(x-a_{2}\right), \ldots$ and so conversely the set of polynomials appropriate to formula (2) and satisfying conditions (2a) is

$\theta_{1}^{-1}\left(x-a_{0}\right)=\left(x-a_{0}\right)\left(x-a_{1}\right),\left(\theta_{2} \theta_{1}\right)^{-1}\left(x-a_{0}\right)=\left(x-a_{0}\right)\left(x-a_{1}\right)\left(x-a_{2}\right), \ldots$

Hence we have

$$
f(x)=f\left(a_{0}\right)+\left(x-a_{0}\right) f\left(a_{0}, a_{1}\right)+\left(x-a_{0}\right)\left(x-a_{1}\right) f\left(a_{0}, a_{1}, a_{2}\right)+\ldots
$$

which is Newton's divided difference formula of interpolation. 
(v) Let $\theta_{1}=\theta_{2}=\ldots=\frac{1}{2}(D+\Delta)=Q$, that is, the operation of forming the mean of a first derivative and a first difference. The inverse operations of formula (1) readily give the polynomials

$$
Q^{-1} x=\left(x^{2}-\frac{1}{2} x\right) / 2 !, Q^{-2} x=\left(x^{3}-\frac{3}{2} x^{2}+\frac{1}{4} x\right) / 3 !, \ldots
$$

and so

$$
f(x)=f(0)+x Q f(0)+\frac{x^{2}-\frac{1}{2} x}{2 !} Q^{2} f(0)+\frac{x^{3}-\frac{3}{2} x^{2}+\frac{1}{4} x}{3 !} Q^{3} f(0)+\ldots
$$

Numerous other operations of a similar kind might be exemplified in this way, both new ones and linear combinations (with non-zero sum of coefficients) of the previous ones, with their appropriate formulae of interpolation, but the above examples and remarks will be sufficient to indicate the nature and wide application of the general formula. 